\title{
Filter-Based Online System-Parameter Estimation for Multicopter UAVs
}

\author{
Christoph Böhm, Martin Scheiber, Stephan Weiss \\ University of Klagenfurt, Austria \\ Email: \{firstname.lastname\}@ieee.org
}

\begin{abstract}
Accurate system modeling and identification gain importance as tasks executed by autonomously acting unmanned aerial vehicles (UAVs) get more complex and demanding.

This paper presents a Bayesian filter approach to online and continuously identify the system parameters, sensor suite calibration states, and vehicle navigation states in a holistic framework. Previous work only tackles subsets of the overall state vector during dedicated phases (e.g., motionless, online during flight, post-processing). These works often introduce the artificial so-called body frame forcing assumptions on system states, such as the inertia matrix's principal axes orientation. Our approach estimates the entire state vector in the (usually not precisely known) center of mass, eliminating several assumptions caused by the artificially introduced body frame in other work. Since our approach also estimates geometric states such as the rotor and sensor placements, no hand-made measures to the unknown center of mass are required - the system is fully self-calibrating. A detailed discussion on the system's observability reveals additionally required (different) measurements for a theoretical and a real $N$-arm multicopter. We show that easy and precise hand-measurable quantities in real applications can provide the required information. Statistically relevant simulations in Gazebo/RotorS providing ground truth for all states yet having realistic physics validate all our findings.
\end{abstract}

\section{INTRODUCTION}

Adaptation to unknown robot configuration changes is significant for easy-to-use, plug-and-play-ready systems usable by anyone. As such, the UAV must be self-aware of all its geometric, inertial, and aerodynamic parameters needed for safe navigation in uncontrolled environments. Knowing these states can yield improved performance in tracking and controlling the UAV using a model-based adaptive scheme.

With this work, we propose and analyze a generalized UAV estimation framework for online system identification, selfcalibration, and localization and show its applicability using a common quadrotor. We can determine nearly all physical states needed for successful flight tracking by using the center of mass as the physical frame of reference instead of the commonly used arbitrary body frame. Relaxing assumptions and constraints compared to the state-of-the-art allows for a more versatile estimation. Easily measurable a priori knowledge of the UAV setup, such as the mass and rotor-to-rotor distances, makes the system fully observable with only a position (or pose) sensor and inertial measurement unit (IMU) measurements available. We support our claims through a series of realistic simulation experiments in Gazebo/RotorS that validate the theoretical result of the nonlinear observability analysis.
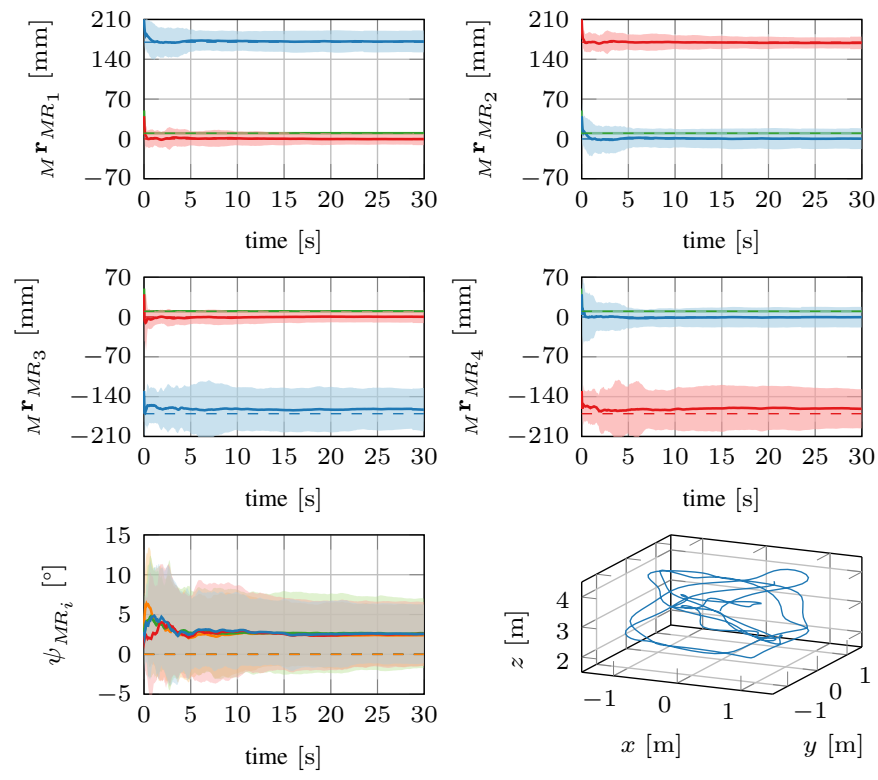

Fig. 1. Estimated rotor displacement ${ }_{M} \mathbf{r}_{M R_{i}}$ and inclination $\psi_{M R_{i}}$ with known $\theta_{M R_{i}}$ based on Lissajous trajectories (e.g., bottom right figure). The solid lines are the mean, the shaded areas the standard deviation, and the dashed lines the ground truth of the respective estimates over 30 test runs. Axis x (blue), y (red), z (green) or rotor 1 (blue), 2 (red), 3 (green), 4 (orange).

This paper presents the following contributions:

- Online estimation of geometric, inertial, and aerodynamic parameters and sensor states for a multicopter UAV including: arm geometry as full 5D rotor placement with 3D translation and 2D thrust/spin direction for each rotor, vehicle mass and moments of inertia, thrust force, and drag moment coefficient for each rotor, IMU biases as well as all sensor extrinsics, and vehicle control states.

- Representation of all states with respect to the vehicle's center of mass and principal axes such that moments of inertia suffice to represent its mass inertia matrix. All transformations with respect to this frame are estimated online to avoid difficult and inaccurate handmeasurements of the potentially changing center of mass.

- Detailed system observability analysis and in-depth discussion on the unobservable dimensions in theory, on differences in practical realizations, and on a generalization to $N$-arm multicopters.

- Validation of the observability analysis and the state estimation with realistic experiments in Gazebo/RotorS. 


\section{RELATED WORK}

Self-calibration with application to an UAV gained attention with the works of Trawny and Roumeliotis [16], Kelly and Sukhatme [9], Weiss [19], and Weiss and Siegwart [20].

All these approaches use either an extended Kalman filter (EKF) variant or an unscented Kalman filter (UKF). IMU measurements as system input replace the underlying system dynamics to simplify the propagation of the estimation. Exteroceptive sensors, e.g., position or pose sensors (visual or other), allow the update of the predicted state estimates. The addition of calibration states such as the transformation between exteroceptive sensors and IMU, IMU biases, or their position on the UAV allows sensor self-calibration during runtime. Although this method makes online state estimation and sensor self-calibration on low-powered hardware possible, it does not use all available information, e.g., the motor speeds available on the flight control unit (FCU). Hence, these approaches lack the capability of online system identification of the underlying physical model.

Using a proper physical model of the UAV leads to improved performance in navigation and control tasks. This statement gets supported by the increase of model-based control schemes used for UAV control: Mellinger et al. [11], Kamel et al. [8], Furrer et al. [5], and Tzoumanikas et al. [17] show such an improvement assuming known system parameters to improve the control performance.

Burri et al. [2], [3], Six et al. [13], and Dhaybi and Daher [4] apply the idea of self-calibration to geometrical, inertial, and aerodynamic properties of the UAV based on offline nonlinear least-squares algorithms. They show that a proper system and sensor model allows the estimation of physical parameters of the UAV's rigid body model. Such algorithms produce better estimation results for the control states but can not be used online on small and computationally limited UAVs due to the problem complexity and data stream length. On the other hand, the proposed filter-based approach allows online on-board estimation in a fixed time through implicit marginalization.

Wuest et al. [21] and Svacha et al. [15] provide physical self-calibration with filter-based approaches, either EKF or UKF. In Wuest et al. [21], the center of mass, the moments of inertia, and the mass of the UAV are part of an EKF and UKF as self-calibration states. Their approach uses rigid body dynamics to model the system behavior in combination with rotor speeds as input. The filter applies corrections with measurements from an IMU and visual inertial odometry (VIO)-based pose sensor. As a result, a change of payload that renders offline calibration values invalid does not influence the task performance. The assumptions on the rotational alignment between reference frames (e.g., the principal axis of inertia matrix/sensor frames and body frame) or the neglecting of translational elements (z-translation between IMU and body frame) reduces the versatility of their self-calibration approach.

However, the work in this paper presents a more generalized approach by reducing those assumptions and adding sensor rotations as well as rotor-related geometric and aerodynamic parameters to the estimation.

The recent work of Svacha et al. [15] shows that an UKF can estimate inertial and aerodynamic properties with IMU and motor speed measurements. It uses the commanded rotor speeds as the system input. The estimation includes the UAV's mass, moments of inertia, horizontal and vertical drag coefficients, and the rotor-motor setup's time-constant and moment of inertia. The authors highlight the need for proper modeling of drag effects at the rotor hub, the rotor's blade flapping moment, and a first-order system model of the rotor-motor setup to improve moments of inertia estimation. The fully observable system presents a reduced state vector containing only a subset of control-related states compared to this work. It also assumes that the rotor thrust and moment coefficients are known and do not change.

We propose to include those states together with rotor displacement and orientation into the run-time estimation process for an increased degree of versatility. We chose an EKF variant over the UKF as it holds reliable fixed time steps even with increasing complexity.

\section{NOTATION}

A leading subscript in front of the variable indicates its reference frame. This work uses $W$ for the fixed world frame, $M$ to denote the center of mass, $P$ as the frame of the exteroceptive sensor (e.g., position or pose), $I$ to label the IMU's frame, and $R_{i}$ for each rotor's frame. The illustration in Fig. 2 shows all these reference frames in context. The variable ${ }_{W} \mathbf{r}_{W M}$ is a position vector pointing from frame $W$ to frame $M$ expressed in the coordinate frame $W$. Therefore, we define a position (and velocity) vector as ${ }_{[\mathrm{Frame}]} \mathbf{r}_{[\mathrm{From}][\mathrm{To}]}$. Quaternions represent the rotation between reference frames. The orientation of $W$ with respect to $M$ is defined as Hamiltonian quaternion $\mathbf{q}_{W M}$, and its rotation matrix $\mathbf{R}_{W M}$ applies to a vector the following way ${ }_{W} \mathbf{r}_{W M}=\mathbf{R}_{W M M} \mathbf{r}_{W M}$. Accordingly, a quaternion is defined as $\mathbf{q}_{[T o][\mathrm{From}]}$. Furthermore, the conjugate of a quaternion is written as $\mathbf{q}_{W M}^{*}$ and corresponds to $\mathbf{R}_{W M}^{\top}$. In this work, we omit the indication of time dependency and write $\mathbf{x}=\mathbf{x}(t)$ to ease the notation and readability.

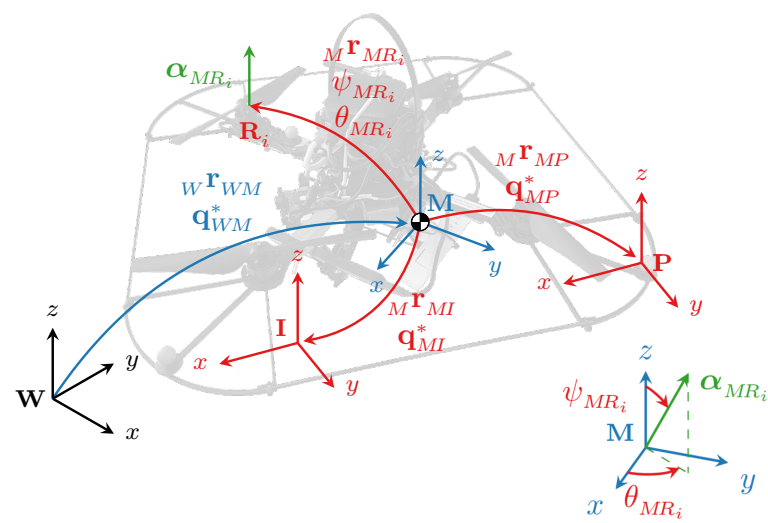

Fig. 2. Reference frames of the proposed UAV model and spherical coordinate definition of the rotor orientation axis $\boldsymbol{\alpha}_{M R_{i}}$. 


\section{System Model of The UAV}

The system model uses the center of mass (CoM) $M$ as the reference frame, in contrast to Burri et al. [3] and Svacha et al. [15], who use a so-called body frame $B$. Using such a body frame seems to be tempting for engineering conveniences as it allows the user to define the location from where one can easily measure, e.g., motor or sensor displacements. However, it is this freedom that introduces unobservable dimensions into the estimation process (e.g., z-translation between $B$ and a sensor), as shown in Böhm et al. [1].

Choosing the CoM as the reference frame is physically a more natural representation but introduces engineering difficulties as this reference frame is usually not precisely known. These difficulties arise when one needs to measure angles and distances of elements (e.g., motors or sensors) with respect to $M$ since the CoM might not be easily or/and accurately calculated for complex UAV configurations. In this work, we show in Section $\nabla$ that we can estimate all necessary variables in a fully self-calibrating system. In particular, we show that, naively, the system would be unobservable, but we can add quantities that are easily measurable on real systems to achieve full observability without the need for a body frame.

\section{A. State Vector}

The trajectory state vector $\mathbf{x}_{T}$ includes ${ }_{W} \mathbf{r}_{W M},{ }_{M} \mathbf{v}_{W M}$, $\mathbf{q}_{W M}$, and ${ }_{M} \boldsymbol{\omega}_{W M}$ which represent the position, linear velocity, orientation, and angular velocity of $M$, respectively.

$$
\mathbf{x}_{T}=\left[{ }_{W} \mathbf{r}_{W M}^{\top},{ }_{M} \mathbf{v}_{W M}^{\top}, \mathbf{q}_{W M}^{\top},{ }_{M} \boldsymbol{\omega}_{W M}^{\top}\right]^{\top} \in \mathbb{R}^{13 \times 1}
$$

Full sensor self-calibration of pose, position, or IMU sensors is possible by adding their displacement and orientation with respect to $M$ to the sensor state vector $\mathbf{x}_{S}$; see Weiss and Siegwart [20] for reference. Hence, ${ }_{M} \mathbf{r}_{M P}$ is the position, and $\mathbf{q}_{M P}$ the rotation quaternion of the exteroceptive sensor $P$. Further, ${ }_{M} \mathbf{r}_{M I}$ expresses the position, and $\mathbf{q}_{M I}$ the rotation quaternion of the IMU $I$. The states ${ }_{I} \mathbf{b}_{a}$ and ${ }_{I} \mathbf{b}_{\omega}$ are the IMU's linear acceleration and angular velocity bias, respectively.

$$
\mathbf{x}_{S}=\left[{ }_{M} \mathbf{r}_{M P}^{\top}, \mathbf{q}_{M P}^{\top},{ }_{M} \mathbf{r}_{M I}^{\top}, \mathbf{q}_{M I}^{\top},{ }_{I} \mathbf{b}_{a}^{\top},{ }_{I} \mathbf{b}_{\omega}^{\top}\right]^{\top} \in \mathbb{R}^{20 \times 1}
$$

$\mathbf{x}_{I}$ contains all inertial properties, such as the mass $m$ of the $\mathrm{UAV}$, the moments of inertia, and the Earth's gravitational pull ${ }_{W} \boldsymbol{g}$ on the on the UAV. The principal axes of the UAV's mass inertia matrix ${ }_{M} \mathbf{I}$ coincide with $M$. As a result, only the diagonal elements ${ }_{M} i$ (moments of inertia) need to be estimated, ${ }_{M} \mathbf{I}=\operatorname{diag}\left({ }_{M} \boldsymbol{i}\right)$. This avoids errors caused by a misalignment between a body frame $B$ and $M$ as it may be present in Burri et al. [2] and Wuest et al. [21].

$$
\mathbf{x}_{I}=\left[m,{ }_{M} \boldsymbol{i}^{\top},{ }_{W} \boldsymbol{g}^{\top}\right]^{\top} \in \mathbb{R}^{7 \times 1}
$$

This work includes the rotor displacements ${ }_{M} \mathbf{r}_{M R_{i}}$ in the geometrical and aerodynamic state vector $\mathbf{x}_{G_{i}}$ per rotor $i$. A full 3D attitude representation of the rotor orientation would result in an unobservability around the motor's spin axis (yaw). Consequently, we chose a minimal attitude representation in spherical coordinates. The inclination $\psi_{M R_{i}}$ measures from the z-axis and the azimuth $\theta_{M R_{i}}$ from the x-axis of $M$, Fig. 2

$$
\boldsymbol{\alpha}_{M R_{i}}=\left[\begin{array}{c}
\sin \psi_{M R_{i}} \cos \theta_{M R_{i}} \\
\sin \psi_{M R_{i}} \sin \theta_{M R_{i}} \\
\cos \psi_{M R_{i}}
\end{array}\right]
$$

The vector $\boldsymbol{\alpha}_{M R_{i}}$ is the resulting thrust/spin axis of the rotor. Thrust force coefficients $k_{f_{i}}$ and drag moment coefficients $k_{m_{i}}$ of each rotor $i$ are also part of $\mathbf{x}_{G_{i}}$. These last-mentioned inclusions to the state vector reduce the amount of a priori assumptions needed during the estimation, compared to Wuest et al. [21] and Tzoumanikas et al. [18].

$$
\mathbf{x}_{G_{i}}=\left[{ }_{M} \mathbf{r}_{M R_{i}}^{\top}, \psi_{M R_{i}}, \theta_{M R_{i}}, k_{f_{i}}, k_{m_{i}}\right]^{\top} \in \mathbb{R}^{7 \times 1}
$$

The complete state vector $\mathrm{x}$ contains then $40+7 N$ elements with $N$ being the number of rotors. Assuming a UAV with $N=4$ rotors, the state vector has a size of 68 elements.

$$
\mathbf{x}=\left[\mathbf{x}_{T}^{\top}, \mathbf{x}_{S}^{\top}, \mathbf{x}_{I}^{\top}, \mathbf{x}_{G_{1}}^{\top}, \ldots, \mathbf{x}_{G_{N}}^{\top}\right]^{\top} \in \mathbb{R}^{(40+7 N) \times 1}
$$

\section{B. System Dynamics}

First-order time-dependent differential equations $\mathbf{f}(\mathbf{x}, \mathbf{u}, \mathbf{w})$ model the UAV's CoM $M$ dynamics. These equations use the current state $\mathbf{x}$, the control input $\mathbf{u}$, and the process noise $\mathbf{w}$. The control input consists of a set of $N$ angular rotor velocities $\omega_{i}, \mathbf{u}=\left[\omega_{1}, \ldots, \omega_{N}\right]^{\top}$.

Regarding the system dynamics, the first step in modeling them is to calculate the force ${ }_{M} \mathbf{F}_{t}$, Eq. (7), and torque ${ }_{M} \mathbf{M}_{t}$, Eq. (9), acting on the UAV's CoM. ${ }_{M} \mathbf{F}_{i}$, Eq. (8), and ${ }_{M} \mathbf{M}_{i}$, Eq. (10), refer to the forces and torques that each rotor generates, respectively.

$$
\begin{aligned}
{ }_{M} \mathbf{F}_{t} & =\sum_{i=1}^{N}{ }_{M} \mathbf{F}_{i} \\
{ }_{M} \mathbf{F}_{i} & =\boldsymbol{\alpha}_{M R_{i}} k_{f_{i}}\left(\omega_{i}+\mathrm{w}_{i}\right)^{2} \\
{ }_{M} \mathbf{M}_{t} & =\sum_{i=1}^{N}\left({ }_{M} \mathbf{M}_{i}+\left[{ }_{M} \mathbf{r}_{M R_{i}}\right]_{\times}{ }_{M} \mathbf{F}_{i}\right) \\
{ }_{M} \mathbf{M}_{i} & = \pm \boldsymbol{\alpha}_{M R_{i}} k_{m_{i}} k_{f_{i}}\left(\omega_{i}+\mathrm{w}_{i}\right)^{2}
\end{aligned}
$$

The noise $\mathrm{w}_{i} \sim \mathcal{N}\left(0, \sigma_{i}^{2}\right)$ models the uncertainty of the commanded rotor speeds sent to the electronic speed controller (ESC). Other external forces and torques caused by aerodynamic effects, e.g., velocity induced hub forces and roll moments, are not modeled. $[\bullet]_{x}$ is the skew-symmetric matrix according to Solà [14]. By combining Eq. (7) and Eq. (9) with Newton-Euler equations and a rigid body assumption, we get the differential equations of $\mathrm{x}_{T}$, Eq. (11) to Eq. (14).

$$
\begin{aligned}
{ }_{W} \dot{\mathbf{r}}_{W M} & =\mathbf{R}_{W M M} \mathbf{v}_{W M} \\
{ }_{M} \dot{\mathbf{v}}_{W M} & =\frac{1}{m}{ }_{M} \mathbf{F}_{t}+\mathbf{R}_{W M W}^{\top} \boldsymbol{g}-\left[{ }_{M} \boldsymbol{\omega}_{W M}\right]_{\times M} \mathbf{v}_{W M} \\
\dot{\mathbf{q}}_{W M} & =\frac{1}{2} \mathbf{q}_{W M} \otimes\left[0,{ }_{M} \boldsymbol{\omega}_{W M}^{\top}\right]^{\top} \\
{ }_{M} \dot{\boldsymbol{\omega}}_{W M} & ={ }_{M} \mathbf{I}^{-1}\left({ }_{M} \mathbf{M}_{t}-\left[{ }_{M} \boldsymbol{\omega}_{W M}\right]_{\times M} \mathbf{I}_{M} \boldsymbol{\omega}_{W M}\right)
\end{aligned}
$$

Eq. (11) refers to the change in position ${ }_{W} \mathbf{r}_{W M}$ caused by the into the world frame $W$ rotated linear velocity ${ }_{M} \mathbf{v}_{W M}$. The linear velocity ${ }_{M} \mathbf{v}_{W M}$ changes, according to Eq. (12), as a result 
TABLE I. Observability analysis of the system model with a state vector size of 68 (number of rotors $N=4$ ). The observability matrix $\mathcal{O}$ shows observable (green) and jointly observable (blue) subspaces depending on the sensor configuration with the other states being independently unobservable (red).

\begin{tabular}{|c|c|c|c|c|c|c|c|c|c|c|c|c|c|c|c|c|c|c|c|}
\hline Measurement & $\begin{array}{c}\text { observable } \\
\text { dimensions }\end{array}$ & ${ }_{W} \mathbf{r}_{W M}$ & ${ }_{M} \mathbf{v}_{W M}$ & $\mathbf{q}_{W M}$ & ${ }_{M} \boldsymbol{\omega}_{W M}$ & ${ }_{M} \mathbf{r}_{M P}$ & $\mathbf{q}_{M P}$ & ${ }_{M} \mathbf{r}_{M I}$ & $\mathbf{q}_{M I}$ & ${ }_{I} \mathbf{b}_{a}$ & ${ }_{I} \mathbf{b}_{\omega}$ & $m$ & ${ }_{M} \boldsymbol{i}$ & ${ }_{W} \boldsymbol{g}$ & ${ }_{M} \mathbf{r}_{M R_{i}}$ & $\psi_{M R_{i}}$ & $\theta_{M R_{i}}$ & $k_{f_{i}}$ & $k_{m_{i}}$ \\
\hline Pose \& IMU & 62 & ok & ok & ok & ok & ok & ok & ok & ok & ok & ok & $\mathrm{J} 2$ & $\mathrm{~J} 3$ & ok & $\mathrm{J} 3$ & ok & ok & J2 & $\mathrm{J} 3$ \\
\hline Position \& IMU & 58 & ok & ok & ok & ok & ok & unobs. & ok & ok & ok & ok & $\mathrm{J} 2$ & $\mathrm{~J} 3$ & ok & $\mathrm{J} 3$ & ok & ok & $\mathrm{J} 2$ & $\mathrm{~J} 3$ \\
\hline Pose & 49 & ok & ok & ok & ok & ok & ok & unobs. & unobs. & unobs. & unobs. & $\mathrm{J} 2$ & $\mathrm{~J} 3$ & ok & $\mathrm{J} 3$ & ok & ok & $\mathrm{J} 2$ & $\mathrm{~J} 3$ \\
\hline Position & 45 & ok & ok & ok & ok & ok & unobs. & unobs. & unobs. & unobs. & unobs. & $\mathrm{J} 2$ & $\mathrm{~J} 3$ & ok & $\mathrm{J} 3$ & ok & ok & $\mathrm{J} 2$ & J3 \\
\hline IMU & 30 & unobs. & unobs. & unobs. & ok & unobs. & unobs. & ok & ok & J1 & ok & $\mathrm{J} 1$ & $\mathrm{~J} 1$ & unobs. & J1 & J1 & $\mathrm{J} 1$ & J1 & $\mathrm{J} 1$ \\
\hline
\end{tabular}

of the force ${ }_{M} \mathbf{F}_{t}$, and accelerations ${ }_{W} \boldsymbol{g}$ and $\left[{ }_{M} \boldsymbol{\omega}_{W M}\right]_{\times M} \mathbf{v}_{W M}$ acting on $M$. Eq. (14) models the dynamics of ${ }_{M} \boldsymbol{\omega}_{W M}$ with Euler's rotation equation around $M$ including the torque ${ }_{M} \mathbf{M}_{t}$ and mass inertia matrix ${ }_{M} \mathbf{I}$. Brownian motion describes the drift of the IMU biases ${ }_{I} \dot{\mathbf{b}}_{a}=\mathbf{w}_{I} \mathbf{b}_{a} \sim \mathcal{N}\left(0, \sigma_{I}^{2} \mathbf{b}_{a}\right)$ and ${ }_{I} \dot{\mathbf{b}}_{\omega}=\mathbf{w}_{I} \mathbf{b}_{\omega} \sim \mathcal{N}\left(0, \sigma_{I}^{2} \mathbf{b}_{\omega}\right)$, respectively. All remaining states of $\mathbf{x}_{S}, \mathbf{x}_{I}$, and $\mathbf{x}_{G_{i}}$ are assumed constant over time.

\section{Measurement Models}

During the estimation process, the state vector updates through comparing pose, position, or/and IMU measurements with measurement models $\mathbf{h}(\mathbf{x}, \mathbf{u}, \mathbf{v})$ based on the current state $\mathbf{x}$, the control input $\mathbf{u}$, and the measurement noise $\mathbf{v}$. Although each sensor might be translated and orientated differently than $M$, they are assumed to be rigidly attached to the CoM.

The sensor model $\mathbf{h}_{\text {imu }}$, Eq. (15), of the IMU assumes that the sensor provides linear acceleration and angular velocity in 3D. Calculating the accelerations at $I$ is possible with the rigid body acceleration ${ }_{M} \mathbf{a}_{a c t}$, and the displacement ${ }_{M} \mathbf{r}_{M I}$. The acceleration ${ }_{M} \mathbf{a}_{\text {act }}$ includes gravity through the thrust force ${ }_{M} \mathbf{F}_{t}$ of the airborne UAV. We do not model external forces acting on the UAV (e.g., wind gusts or normal force when landed). ${ }_{M} \dot{\boldsymbol{\omega}}_{W M}$ is modelled through Eq. (14). Further, ${ }_{M} \boldsymbol{\omega}_{W M}$ is used as the rigid body assumption makes angular velocities on the whole system equal, ${ }_{M} \boldsymbol{\omega}_{W M} \equiv{ }_{M} \boldsymbol{\omega}_{W I}$. The angular velocity model uses the state ${ }_{M} \boldsymbol{\omega}_{W M}$. Rotation matrix $\mathbf{R}_{M I}^{\top}$ rotates both models into the IMU frame $I$. Both measurements are subject to measurement bias ${ }_{I} \mathbf{b}_{a}$ and ${ }_{I} \mathbf{b}_{\omega}$ as well as noise $\mathbf{v}_{a} \sim \mathcal{N}\left(0, \sigma_{a}^{2}\right)$ and $\mathbf{v}_{\omega} \sim \mathcal{N}\left(0, \sigma_{\omega}^{2}\right)$.

$$
\begin{aligned}
\mathbf{h}_{i m u}= & {\left[\begin{array}{c}
\mathbf{R}_{M I M}^{\top} \mathbf{a}_{a c t}+{ }_{I} \mathbf{b}_{a}+\mathbf{v}_{a} \\
\mathbf{R}_{M I M}^{\top} \boldsymbol{\omega}_{W M}+{ }_{I} \mathbf{b}_{\omega}+\mathbf{v}_{\omega}
\end{array}\right], } \\
{ }_{M} \mathbf{a}_{a c t}= & \frac{1}{m}{ }_{M} \mathbf{F}_{t}+\left[{ }_{M} \dot{\boldsymbol{\omega}}_{W M}\right]_{\times M} \mathbf{r}_{M I} \\
& +\left[{ }_{M} \boldsymbol{\omega}_{W M}\right]_{\times}\left(\left[{ }_{M} \boldsymbol{\omega}_{W M}\right]_{\times M} \mathbf{r}_{M I}\right)
\end{aligned}
$$

As pose sensor, we assume a sensor that provides the estimation with absolute position and orientation of the sensor $P$ with respect to $W$. Whereas a position sensor, e.g., a global positioning system (GPS) receiver, can only provide absolute position information. The pose measurement $\mathbf{h}_{\text {pose }}$, Eq. (17), is a result of the coordinate transformation from $M$ to the sensor $P$ with ${ }_{M} \mathbf{r}_{M P}$ and $\mathbf{q}_{M P}$, respectively. The noise terms $\mathbf{v}_{p} \sim \mathcal{N}\left(0, \sigma_{p}^{2}\right)$ and $\mathbf{v}_{\theta} \sim \mathcal{N}\left(0, \sigma_{\theta}^{2}\right)$ depend on the sensor used and are assumed to be independent.

$$
\mathbf{h}_{\text {pose }}=\left[\begin{array}{c}
{ }_{W} \mathbf{r}_{W M}+\mathbf{R}_{W M M} \mathbf{r}_{M P}+\mathbf{v}_{p} \\
\mathbf{q}_{W M} \otimes \mathbf{q}_{M P} \otimes \mathbf{v}_{q}
\end{array}\right], \mathbf{v}_{q}=\left[\begin{array}{c}
1 \\
\frac{1}{2} \mathbf{v}_{\theta}
\end{array}\right]
$$

\section{NONLINEAR OBSERVABILITY ANALYSIS}

The observability matrix $\mathcal{O}$ of the system model from Sec. IV shows with its rank and in its null-space observable and unobservable subspaces of the state space as well as jointly observable states, Hermann and Krener [7], Kelly and Sukhatme [9], and Martinelli [10].

$$
\mathcal{O}(\mathbf{x}, \mathbf{u})=\left[\left(\nabla L^{0} \mathbf{h}\right)^{\top},\left(\nabla L_{\mathbf{f}_{0}}^{1} \mathbf{h}\right)^{\top},\left(\nabla L_{\mathbf{f}_{1}}^{1} \mathbf{h}\right)^{\top}, \ldots\right]^{\top},
$$

with $L^{0} \mathbf{h}=\mathbf{h}(\mathbf{x}, \mathbf{u})$ and $L_{\mathbf{f}}^{i} \mathbf{h}=\frac{\partial\left(L_{\mathbf{f}}^{i-1} \mathbf{h}\right)}{\partial \mathbf{x}} \mathbf{f}(\mathbf{x})$. We use the control-affine form of $\mathbf{f}$ and a variable substitution $\mathbf{u}^{*}=\left[\omega_{1}^{2}, \ldots, \omega_{N}^{2}\right]^{\top}$ of the squared rotor speeds for the Lie derivatives. Additional measurements ensure that the unit length constraint holds for rotation quaternions.

$$
\mathbf{h}_{\text {unit }}=\left[\mathbf{q}_{W M}^{\top} \mathbf{q}_{W M}, \mathbf{q}_{M P}^{\top} \mathbf{q}_{M P}, \mathbf{q}_{M I}^{\top} \mathbf{q}_{M I}\right]^{\top}
$$

\section{A. Observability Discussion}

The analysis of the observability matrix $\mathcal{O}$ follows two steps, a symbolic and numerical rank calculation as well as a numerical study of its null-space. In the general observability analysis, all state values and control inputs are random values within the same order of magnitude. This avoids ill-conditioned problem sets as it may occur with real system values (e.g., UAV position value is several orders of magnitude larger than rotor thrust force coefficients, cf. Sec. V-B. Rotations are set to random values through their quaternions and angles, respectively. The results presented in Tab. I assume a quadrotor system $(N=4)$. However, the observability analysis was done with one, four, six, and eight rotor configurations to show the scalability of the approach.

1) Pose \& IMU: With pose and IMU measurements available, the observability matrix $\mathcal{O}$ has a rank of 62 of 68 in the case of four rotors. Consequently, $\mathcal{O}$ has six unobservable dimensions. This six-dimensional subspace spans over the 24 states $m,{ }_{M} \boldsymbol{i},{ }_{M} \mathbf{r}_{M R_{i}}, k_{f_{i}}$, and $k_{m_{i}}$. All other states are immediately observable (44 dimensions). One unobservable dimension, $J 2$, is a joint observability between $m$ and $k_{f_{i}}$. J2 is the result of the thrust force coefficients and mass being in a ratio to each other, Eq. (8) and Eq. (12). Hence, an estimate of the relation between those states is feasible due to Eq. (17) but lacks absolute information. Furthermore, this one-dimensional subspace $J 2$ remains even with a changing number of rotors.

The remaining five dimensions are part of the subspace $J 3$ that spans over ${ }_{M} \boldsymbol{i},{ }_{M} \mathbf{r}_{M R_{i}}$, and $k_{m_{i}}$. It resembles the ambiguity of the rotor geometry and its influence on the rotational dynamics. Different values of ${ }_{M} \boldsymbol{i},{ }_{M} \mathbf{r}_{M R_{i}}$, and $k_{m_{i}}$ can result in 
the same change of angular velocity ${ }_{M} \dot{\boldsymbol{\omega}}_{W M}$, as it contributes to Eq. (9) and Eq. (14). Knowledge of the Euclidean distance between any pair of rotors, $\left\|_{M} \mathbf{r}_{M R_{j}}-{ }_{M} \mathbf{r}_{M R_{i}}\right\|_{2}, i \neq j$ (i.e. adding this information as a measurement in an estimator), makes a single dimension out of the five observable. Therefore, knowing five of six possible rotor-to-rotor distances in a quadcopter setup makes all five unobservable dimensions in $J 3 \mathrm{ob}$ servable. $J 3$ scales linearly with the number of rotors $N$ used and has thus $1+N$ unobservable dimensions. Interestingly, this means that these rotor-to-rotor measurements cannot make one-, two-, and three-rotor configurations observable. Thus, one can infer that the state vector's observable dimensions scale with $38+6 N$ without any additional assumptions.

2) Position \& IMU: This measurement configuration is of interest as it resembles most of the outdoor UAV setups. Such UAVs are often equipped with a global navigation satellite system (GNSS) measurement sensors, e.g., a GPS receiver, and an IMU. Interestingly, we see a similar result to Sec. V-A1 suggesting that, if we have motion, the attitude part in Sec. V-A1 does, theoretically, not add new information. Naturally, zerodynamics in $\mathbf{f}$ and the absence of absolute attitude information from Eq. (17) make $\mathbf{q}_{M P}$ unobservable, stating that the attitude between the GNSS sensor and $M$ is irrelevant. The analysis shows that the angular velocity ${ }_{M} \boldsymbol{\omega}_{W M}$ is observable even without IMU measurements, only improving the estimation quality. Looking at different rotor numbers reveals that this observation holds for all of them, giving us $34+6 N$ observable dimensions without any additional assumptions.

3) Pose or Position only: Both of those configurations show the same observable and jointly observable states as the ones including an IMU besides all self-calibration states of the IMU. They are unobservable because of the lack of information from Eq. (15). This causes no problem as these states can be disregarded without the presence of an IMU. Interestingly, as mentioned above, the sole position sensor setup can estimate the UAV's world attitude (given enough movement). Once more, these results hold for all considered rotor configurations. Therefore, the number of observable dimensions for single pose or position measurements is $25+6 N$ or $21+6 N$ without any additional assumptions, respectively.

4) IMU only: The number of observable dimensions decreases drastically if the estimation has only access to IMU measurements. As expected, states that rely on pose or position information become unobservable. As a result ${ }_{W} \mathbf{r}_{W M},{ }_{W} \mathbf{v}_{W M}$, $\mathbf{q}_{W M},{ }_{M} \mathbf{r}_{M P}, \mathbf{q}_{M P}$, and ${ }_{W} \boldsymbol{g}$ are unobservable. The IMU measurement, Eq. [15], makes ${ }_{M} \boldsymbol{\omega}_{W M}$ and most of the IMU's self-calibration states observable. The states ${ }_{I} \mathbf{b}_{a}, \psi_{M R_{i}}$, and $\theta_{M R_{i}}$ merge with $J 2$ and $J 3$ into $J 1$ which spans $2+4 N$ unobservable dimensions for an arbitrary number of rotors.

\section{B. AscTec Hummingbird Model}

The observability matrix $\mathcal{O}$ shows different observable, unobservable, and jointly observable dimensions depending on the system configuration. Additionally, as some states may be several orders of magnitude larger than others, the estimator may face ill-conditioned situations leading to numerical issues on real systems when extracting information for the theoretically observable dimensions. The experiments in Sec. VI use the model of an AscTec Hummingbird quadrotor with pose and IMU measurements, system parameters listed in Tab. II

The difference to the general case is that the rotor rotation axes $\boldsymbol{\alpha}_{M R_{i}}$ align with the z-axis of the CoM $M, \psi_{M R_{i}}=0$. Thus, the sine entries in the first and second row of $\boldsymbol{\alpha}_{M R_{i}}$ are zero, Eq. (4). Consequently, the azimuths $\theta_{M R_{i}}$ become unobservable, adding $N$ unobservable dimensions. The skewsymmetric matrix in Eq. (10) of ${ }_{M} \mathbf{M}_{i}$ makes the z-component of the rotor displacements ${ }_{M} \mathbf{r}_{M R_{i}, z}$ unobservable, replacing four dimensions in $J 3$ with purely unobservable ones.

Additionally, the setup's rotor displacements ${ }_{M} \mathbf{r}_{M R_{i}}$ are symmetric along the $\mathrm{x}$ - and $\mathrm{y}$-axis of $M$ and cause $J 3$ to only span one unobservable dimension. This dimension, again, lacks one rotor-to-rotor distance information. One unobservable dimension, $J 2$, spans across $m$ and $k_{f_{i}}$, the same way as for the general case. An additional one-dimensional subspace $J 4$ is the result of the setup at use and contains $\mathbf{q}_{W M, z},{ }_{M} \boldsymbol{\omega}_{W M, x y},{ }_{M} \mathbf{r}_{M P, x y}, \mathbf{q}_{M P, z},{ }_{M} \mathbf{r}_{M I, x y}$, and $\mathbf{q}_{M I, z}$. The relationship of these states to one another is known, but one absolute piece of information needs to be known for a successful estimation. This results in a rank of 57 of 68 , compared to the rank of 62 of the general analysis.

Rearranging the rotor displacements to model a one, six, and eight rotor configuration with given parameters shows that the unobservable dimensions scale linearly with the number of rotors. These results show that UAVs with similar geometric constraints (alignment of thrust axis) as the AscTec Hummingbird quadrotor yield $37+5 \mathrm{~N}$ observable dimensions. However, these jointly observable dimensions enforce applicationspecific assumptions about the system.

\section{EXPERIMENTAL RESULTS}

The proposed system model and its observability analysis are evaluated through realistic simulation experiments with a filter-based implementation and the AscTec Hummingbird model, Tab. II] in Gazebo/RotorS [5].

\section{A. Filter-based Estimation}

We use the iterative error-state Kalman filter (IEKF) implementation in Matlab, as mentioned in Sec. II] as it improves accuracy while still allowing on-board execution on the UAV. The authors refer to Trawny and Roumeliotis [16], Weiss [19], and Solà [14] for a more thorough description of the errorstate representation. According to Sec. V-B, it is assumed that both pose sensor and IMU measurements are present during the estimation. A fully observable system for the AscTec Hummingbird model is achieved by adding measurements for $m$ to make $J 2$ observable, $\theta_{M R_{i}}$ and ${ }_{M} \mathbf{r}_{M R_{i}, z}$ as both of them are unobservable for each rotor, $\left\|_{M} \mathbf{r}_{M R_{2}}-{ }_{M} \mathbf{r}_{M R_{1}}\right\|_{2}$ for the one unobservable dimension of $J 3$, and $\mathbf{q}_{M P z}$ required by $J 4$ (cf. Sec. V-B). All these parameters can be measured before take-off. In the following experiments, the initial state values of the IEKF are approximately $10 \%$ off compared to ground truth to show the convergence of the observable states. 


\section{B. Gazebo/RotorS \& Control}

The above-stated model of the AscTec Hummingbird UAV in the Gazebo/RotorS framework (Furrer et al. [5]) is used to test the IEKF implementation. It models noise and physics realistically such that the evaluation of the estimator is best feasible. The advantage of the Gazebo/RotorS simulation is that most states are known or can be calculated from simulation properties, like the moments of inertia ${ }_{M} i$. These calculations may show only negligible offsets to the real values due to differently modeled effects, e.g., moments of inertia of the spinning rotors. The drag force and rolling moment coefficients (velocity induced hub forces and roll moments on the rotors) of the RotorS model are set to zero to reduce errors due to unmodeled effects. For very fast flights, these effects will start having some effects in the estimation process.

The bias and noise values applied to the IEKF, Tab. II and Tab. III respectively, are taken from multiple time-series of real hardware measurements. An angular bias ${ }_{I} \mathbf{b}_{\omega}$ is not applied as the FCU on the Hummingbird compensates this during the startup sequence. The noise values of the pose sensor assume an absolute tracking system like OptiTrack. Each noise source mentioned is assumed to be zero-mean Gaussian white noise. The frequency at which the FCU and Gazebo publish the motor speed values and IMU measurements is set to $200 \mathrm{~Hz}$, with pose measurements published at $50 \mathrm{~Hz}$. An aggressively tuned model predictive control (MPC) of Kamel et al. [8] allows the flight of trajectories with sufficient excitation in all 6 degrees of freedom (DoF).

This work uses Lissajous figure-based trajectories similar to the ones used by Svacha et al. [15], combining a lowfrequency high-velocity and a high-frequency low-velocity motion, Eq. 20. The values of $c_{j}$ are uniformly distributed,

TABLE II. Gazebo/RotorS AscTec Hummingbird Model Parameters.

\begin{tabular}{clclc}
\hline & x/roll & y/pitch/value & z/yaw & Unit \\
\hline${ }_{M} \mathbf{r}_{M P}$ & $2.6 \cdot 10^{1}$ & $3.8 \cdot 10^{1}$ & $5.9 \cdot 10^{1}$ & $\mathrm{~mm}$ \\
$\mathbf{q}_{M P}$ & 0.0 & 0.0 & 0.0 & $\circ$ \\
${ }_{M} \mathbf{r}_{M I}$ & $1.9 \cdot 10^{1}$ & -9.3 & 3.0 & $\mathrm{~mm}$ \\
$\mathbf{q}_{M I}$ & 0.0 & 0.0 & 0.0 & $\circ$ \\
${ }_{I} \mathbf{b}_{a}$ & $-2.2 \cdot 10^{-1}$ & $-2.1 \cdot 10^{-1}$ & $1.4 \cdot 10^{-1}$ & $\mathrm{~m} / \mathrm{s}^{2}$ \\
${ }_{I} \mathbf{b}_{\omega}$ & 0.0 & 0.0 & 0.0 & $\mathrm{rad} / \mathrm{s}$ \\
\hline$m$ & & $7.2 \cdot 10^{-1}$ & & $\mathrm{~kg}$ \\
${ }_{M} \boldsymbol{i}$ & $7.5 \cdot 10^{-3}$ & $7.5 \cdot 10^{-3}$ & $1.3 \cdot 10^{-2}$ & $\mathrm{~kg} \mathrm{~m}$ \\
${ }_{W} \boldsymbol{g}$ & 0.0 & 0.0 & -9.81 & $\mathrm{~m} / \mathrm{s}^{2}$ \\
\hline${ }_{M} \mathbf{r}_{M R_{1}}$ & $1.7 \cdot 10^{2}$ & 0.0 & $1.1 \cdot 10^{1}$ & $\mathrm{~mm}$ \\
${ }_{M} \mathbf{r}_{M R_{2}}$ & 0.0 & $1.7 \cdot 10^{2}$ & $1.1 \cdot 10^{1}$ & $\mathrm{~mm}$ \\
${ }_{M} \mathbf{r}_{M R_{3}}$ & $-1.7 \cdot 10^{2}$ & 0.0 & $1.1 \cdot 10^{1}$ & $\mathrm{~mm}$ \\
${ }_{M} \mathbf{r}_{M R_{4}}$ & 0.0 & $-1.7 \cdot 10^{2}$ & $1.1 \cdot 10^{1}$ & $\mathrm{~mm}$ \\
$\psi_{M R_{1 \ldots 4}}$ & & 0.0 & & $\circ$ \\
$\theta_{M R_{1 \ldots 4}}$ & & 0.0 & & $\circ$ \\
$k_{f_{1 \ldots 4}}$ & & $3.4 \cdot 10^{-4}$ & & $\mathrm{~N} / \mathrm{s}^{-2}$ \\
$k_{m_{1} \ldots 4}$ & & $1.6 \cdot 10^{-2}$ & & $\mathrm{~m}$ \\
\hline & & & &
\end{tabular}

TABLE III. Gazebo/RotorS AscTec Hummingbird Noise Parameters.

\begin{tabular}{ccccccc}
\hline$\sigma_{i}$ & $\begin{array}{c}\sigma_{I} \mathbf{b}_{a} \\
\mathrm{~m} / \sqrt{\mathrm{s}^{5}}\end{array}$ & $\begin{array}{c}\sigma_{I} \mathbf{b}_{\omega} \\
\mathrm{rad} / \sqrt{\mathrm{s}^{3}}\end{array}$ & $\begin{array}{c}\sigma_{a} \\
\mathrm{~m} / \mathrm{s}^{2}\end{array}$ & $\begin{array}{c}\sigma_{\omega} \\
\mathrm{rad} / \mathrm{s}\end{array}$ & $\begin{array}{c}\sigma_{p} \\
\mathrm{~m}\end{array}$ & $\begin{array}{c}\sigma_{\theta} \\
\mathrm{rad}\end{array}$ \\
\hline 0.5 & $8.3 \cdot 10^{-3}$ & $1.3 \cdot 10^{-4}$ & 0.83 & 0.013 & $1 \cdot 10^{-3}$ & $1.7 \cdot 10^{-3}$ \\
\hline
\end{tabular}

$c_{j} \sim \mathcal{U}(3,8)$, and chosen at random for each trajectory. An example of such a trajectory can be found in Fig. 1. Alternatively, the work of Hausman et al. [6], Preiss et al. [12], and Böhm et al. [1] show that a more elaborated observability-aware optimization strategy on the Eigenvalues of the E ${ }^{2}$ LOG further improves the convergence of self-calibration estimates. In this work, Lissajous trajectories are used because of computation time restrictions during the trajectory generation.

$$
\begin{gathered}
y_{j}(t)=\sin \left(c_{j} \frac{2 \pi t}{t_{\text {end }}}\right)+\frac{1}{10} \sin \left(5 c_{j} \frac{2 \pi t}{t_{\text {end }}}\right), \\
j \in\{x, y, z, y a w\}, c_{j} \in \mathbb{R}
\end{gathered}
$$

\section{Discussion}

We tested the observability empirically on 30 different 30-second Lissajous trajectories to prevent trajectory-induced biases on the estimates. An average run-time of the estimation of 18.8 seconds in Matlab on an i7-7820HQ CPU puts this approach in the real-time application range. The evaluation is based on the convergence behavior seen in the respective figures and the mean and standard deviation of the error L2norm $\|\hat{\mathbf{x}}-\mathbf{x}\|_{2}$ of the estimates $\hat{\bullet}$ compared to ground truth at the end of the trajectory.

Fig. 1 and Fig. 3 show the resulting estimates based on the fully observable system from Sec. V-B and Sec. VI-A The trajectory state vector $\mathbf{x}_{T}$ is not shown in these figures as each trajectory performs a different motion. All states converge fast to an appropriate value within a few seconds, even with a wrong initial guess. This behavior confirms the theoretical observability results from Sec. V] Tab. IV lists all empirical results gathered from all 30 test runs.

1) Sensor States: The self-calibration states ${ }_{M} \mathbf{r}_{M P}$ show a relative error below $4 \%$ on each axis with $\mathbf{q}_{M P}$ errors of less than $1^{\circ}$. Wuest et al. [21] achieved errors in the range of $15 \%$ to $30 \%$ for the pose sensor's displacement state, neglecting its orientation. The least-squares algorithm of Burri et al. [3] performed well with position errors under $8 \%$ and orientation errors below $1^{\circ} \cdot{ }_{M} \mathbf{r}_{M I}$ is a difficult state to estimate with maximum $14 \%$ in $\mathrm{x}$ - and $\mathrm{y}$-direction and almost $86 \%$ in $\mathrm{z}$ direction. This high relative error is in absolute error terms only $2.6 \mathrm{~mm}$ and due to the small distance of the IMU to the CoM resulting in less information through the short lever-arm. Additionally, the presented approach allows the estimation of the IMU's attitude within $1^{\circ}$. In comparison, Wuest et al. [21] achieved a IMU position estimate for $\mathrm{x}$ - and $\mathrm{y}$-axis only based on the EKF between $16 \%$ to $23 \%$. They apply the assumption that the pose sensor and IMU are aligned along the z-axis and therefore do not need to estimate this value. It can be seen that the acceleration bias ${ }_{I} \mathbf{b}_{a}$ is the most difficult one to estimate of the sensor states. On the other hand, the gyroscopic measurement bias ${ }_{I} \mathbf{b}_{\omega}$ converges fast and accurate. 
2) Inertial States: Mass $m$ is excluded from the evaluation as it is assumed to be known a priori mitigating the one unobservable dimension discussed of $J 2$. The moments of inertia ${ }_{M} i$ converge close to ground truth with the z-component having a non-negligible offset. Since this is consistent through all the experiments, we assume that ground truth might also be miscalculated and is subject to further investigation. In addition, low angular velocity values in ${ }_{M} \boldsymbol{\omega}_{W M}$ around the z-axis cause a lower quality of the estimate in ${ }_{M} \boldsymbol{i}_{z}$. This can be seen in the wider z-component spread. As Burri et al. [3] noticed as well, one can see a coupling with moment coefficients $k_{m_{i}}$ as these are the major contributors to yaw motion. ${ }_{M} i$ has a relative error of around $1 \%$ along the $\mathrm{x}$ and $\mathrm{y}$-axis and below $8 \%$ along the $\mathrm{z}$-axis. In Wuest et al. [21] they were able to estimate the moments of inertia below $20 \%$ relative error. Svacha et al. [15] got similar results in the region of $5 \%$ to $22 \%$. The offline approach from Burri et al. [3] states an inertia estimation error under $5 \%$. The gravity vector is converging fast and accurate.

3) Geometrical \& Aerodynamic States: The performance of the rotor displacement estimates ${ }_{M} \mathbf{r}_{M R_{i}}$ cannot be compared to previous works, as they have never been, to the authors' knowledge, included in such an estimation process. Besides that, we are able to estimate the distance to the CoM $M$ with a relative error of $1 \%$ or an absolute error of $9 \mathrm{~mm}$, respectively. The lower standard deviation seen in Fig. 1 for ${ }_{M} \mathbf{r}_{M R_{1}}$ and ${ }_{M} \mathbf{r}_{M R_{2}}$ are due to the additional knowledge of the respective rotor-to-rotor distance $\left\|_{M} \mathbf{r}_{M R_{2}}-{ }_{M} \mathbf{r}_{M R_{1}}\right\|_{2}$, Sec. V-B The inclination angles converge with slight offset as the $\sin \psi_{M R_{i}}$ is very small around zero. Nonetheless, it converges to an absolute error of less than $3^{\circ}$. The thrust force coefficients $k_{f_{i}}$ and the drag moment coefficients $k_{m_{i}}$ show a relative error of maximum $3 \%$ and $12 \%$, respectively. It is worth mentioning that the approach is capable of similar results compared to Burri et al. [3] which was able to estimate the drag moment coefficient below $10 \%$ relative error. The drag moment coefficients exhibit a higher standard deviations because of the limited yaw motions possible with the system.

These results show that the proposed approach is on the same performance level as other works with less states and more assumptions and/or constraints.

4) Joint Observability: Fig. 4 shows the impact on the estimation if the assumption that the mass $m$ is known does not apply during the estimation process. The joint observability $J 2$ is present in both left figures as shared drift between the UAV's mass $m$ and the individual rotor's thrust force coefficients $k_{f_{i}}$. A wrong initial guess in $m$ and $k_{f_{i}}$ will not converge to the ground truth. The moments of inertia ${ }_{M} i$ and drag moment coefficients $k_{m_{i}}$ on the right half of Fig. 4 emphasize that $J 2$ and $J 3$ are independent joint observabilites.

Fig. 5 displays the influence of $J 3$ and the unobservable z-components of the rotor displacements ${ }_{M} \mathbf{r}_{M R_{i}}$ on the estimates. J3 causes a diverging behavior of non-zero ${ }_{M} \mathbf{r}_{M R_{i}, x y}$ elements, moments of inertia ${ }_{M} \boldsymbol{i}$, and drag moment coefficients $k_{m_{i}}$ on all 30 test runs indicated by the spread. The unobservable dimensions of ${ }_{M} \mathbf{r}_{M R_{i}, z}$ are a result of the rotor's
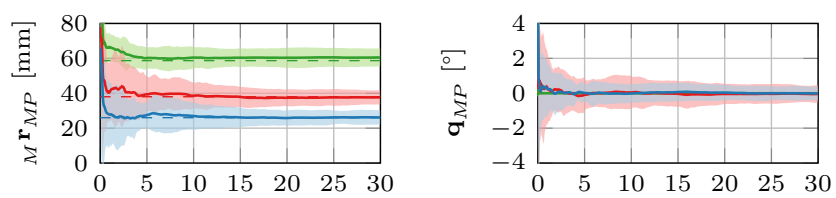

time $[\mathrm{s}]$
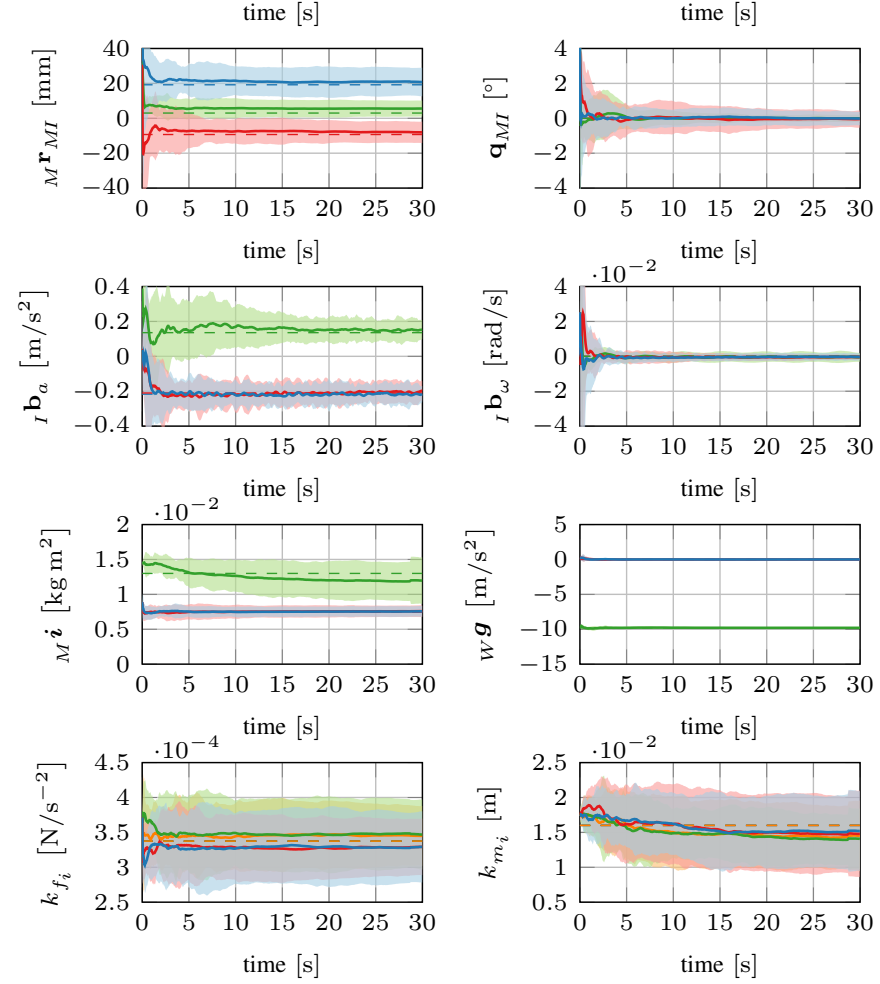

Fig. 3. Estimated sensor, inertial, and aerodynamic parameters based on Lissajous trajectories. Known values of $m$ and $\theta_{M R_{i}}$ are not shown. The solid lines are the mean, the shaded areas the standard deviation, and the dashed lines the ground truth of the respective estimates over 30 test runs. Axis x (blue), y (red), z (green) or rotor 1 (blue), 2 (red), 3 (green), 4 (orange).

TABLE IV. Results of the fully observable system shown through the L2-norm $\|\hat{\mathbf{x}}-\mathbf{x}\|_{2}$ of each state. $m$ and $\theta_{M R_{i}}$ are not listed as those parameters are assumed known.

\begin{tabular}{clcc}
\hline & Absolute Error & Standard Deviation & Unit \\
\hline${ }_{M} \mathbf{r}_{M P}$ & 1.8 & 2.6 & $\mathrm{~mm}$ \\
$\mathbf{q}_{M P}$ & $4.0 \cdot 10^{-2}$ & $2.1 \cdot 10^{-1}$ & $\circ$ \\
${ }_{M} \mathbf{r}_{M I}$ & 3.3 & 3.7 & $\mathrm{~mm}$ \\
$\mathbf{q}_{M I}$ & $4.5 \cdot 10^{-2}$ & $2.2 \cdot 10^{-1}$ & $\circ$ \\
${ }_{I} \mathbf{b}_{a}$ & $2.1 \cdot 10^{-2}$ & $3.0 \cdot 10^{-2}$ & $\mathrm{~m} / \mathrm{s}^{2}$ \\
${ }_{I} \mathbf{b}_{\omega}$ & $7.8 \cdot 10^{-4}$ & $1.2 \cdot 10^{-3}$ & $\mathrm{rad} / \mathrm{s}$ \\
${ }_{M} \boldsymbol{i}$ & $1.0 \cdot 10^{-3}$ & $1.2 \cdot 10^{-3}$ & $\mathrm{~kg} \mathrm{~m}{ }^{2}$ \\
${ }_{W} \boldsymbol{g}$ & $1.5 \cdot 10^{-2}$ & $1.9 \cdot 10^{-2}$ & $\mathrm{~m} / \mathrm{s}^{2}$ \\
${ }_{M} \mathbf{r}_{M R_{1}}$ & 1.4 & 7.3 & $\mathrm{~mm}$ \\
${ }_{M} \mathbf{r}_{M R_{2}}$ & 1.3 & 7.1 & $\mathrm{~mm}$ \\
${ }_{M} \mathbf{r}_{M R_{3}}$ & 7.1 & $1.3 \cdot 10^{1}$ & $\mathrm{~mm}$ \\
${ }_{M} \mathbf{r}_{M R_{4}}$ & 8.6 & $1.3 \cdot 10^{1}$ & $\mathrm{~mm}$ \\
$\psi_{M R_{1 \ldots 4}}$ & 5.1 & 2.6 & $\circ$ \\
${ }_{{ }_{f_{11.4}}}$ & $1.7 \cdot 10^{-5}$ & $3.1 \cdot 10^{-5}$ & $\mathrm{~N} / \mathrm{s}^{-2}$ \\
$k_{m_{1 \ldots 4}}$ & $2.9 \cdot 10^{-3}$ & $3.6 \cdot 10^{-3}$ & $\mathrm{~m}$ \\
\hline & & &
\end{tabular}


alignment with the CoM's z-axis, prohibiting the convergence of these states. Again, a wrong initial guess of those states will not converge to the ground truth. Fig. 5 confirms that zero value components of ${ }_{M} \mathbf{r}_{M R_{i} x y}$ are observable and converge to ground truth, as stated in Sec. V-B.

5) Tilted Rotors: Repeating the 30 test runs with the Hummingbird simulation model having tilted rotors proves the validity of the claim that the rotor's spin axis $\boldsymbol{\alpha}_{M R_{i}}$ is observable in the general case. For this experiment, an inclination angle $\psi_{M R_{i}}$ of $20^{\circ}$ is applied to each rotor. The azimuth angles $\theta_{M R_{i}}$ align the x-axes of $\boldsymbol{\alpha}_{M R_{i}}$ with the respective rotor arm $\left(0^{\circ}, 90^{\circ}, 180^{\circ}\right.$, and $270^{\circ}$, respectively). Fig. 6 shows the result of this experiment and that a nonzero inclination angle makes the rotor spin axis observable as claimed in Sec. V-A. The offset in the estimate of $\psi_{M R}$ is due to the weak contribution of it to the spin axis, Eq. (4).

\section{CONCLUSION}

This paper presented a holistic Bayesian filter approach that allows online estimation of geometric, inertial, aerodynamic, and sensor parameters of a multicopter UAV with $N$-arms.

One insight of this research is that the use of the socalled body frame seems to be tempting for engineering conveniences, but it introduces unobservable dimensions into the estimation process. Therefore, the proposed system model defines the center of mass $M$ aligned with the UAV's inertia matrix principal axes as its reference frame. The addition of a 5D rotor placement through a 3D translation ${ }_{M} \mathbf{r}_{M R_{i}}$ and a 2D thrust/spin direction using spherical coordinates $\left(\psi_{M R_{i}}, \theta_{M R_{i}}\right)$ combined with aerodynamic parameters of the rotor $\left(k_{f_{i}}, k_{m_{i}}\right)$ allows for a new level of self-awareness on UAV multicopter.

Another major part is the observability of the newly added parameters. Hence, a thorough observability analysis of the proposed system model has been conducted in theory, on a practical realization, and is generalized to $N$-arm multicopters to show its versatility. Different measurement configurations (e.g., GPS and IMU) were discussed to identify observable, unobservable, and jointly observable dimensions in the state space. Significant findings are: the world referenced attitude of $M$ is observable through proper motion even with only position measurements, and jointly observable dimensions (e.g., between $m$ and $k_{f_{i}}$ ) arise depending on the application.

The experimental validation was done with an IEKF implementation in Matlab and the simulation environment Gazebo/RotorS, which has the UAV as a model available. The empirical results confirm the insights from the observability analysis, and the estimation results can compete with state-ofthe-art frameworks where comparisons are possible. State-ofthe-art calibration methods with parameters based on the body frame struggle to achieve comparable results to ours.

The inertial, aerodynamic, and sensor states achieved approximately $24 \%$ better estimation accuracy compared to the state-of-the-art. Comparing the estimation performance of the geometric states of each rotor was not possible since, to the authors' knowledge, no approach currently estimates these system parameters.


Fig. 4. Estimation without a priori knowledge of the state $m$. The one unobservable dimension of $J 2$ gets spanned by $m$ and $k_{f_{i}}$ and shows as overall drift. ${ }_{M} i$ and $k_{m_{i}}$ show that $J 2$ has no influence on $J 3$. The solid lines are the mean, the shaded areas the standard deviation, and the dashed lines the ground truth of the respective estimates over 30 test runs. Axis $\mathrm{x}$ (blue), y (red), z (green) or rotor 1 (blue), 2 (red), 3 (green), 4 (orange).
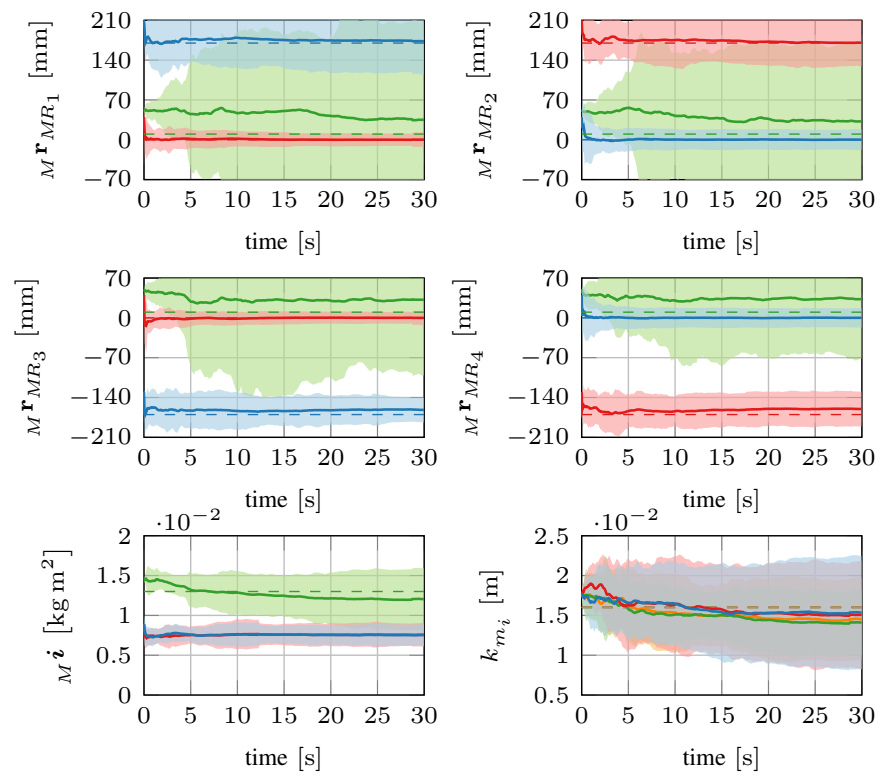

Fig. 5. Estimation without a priori knowledge of the state ${ }_{M} \mathbf{r}_{M R_{i}, z}$ and $\left\|_{M} \mathbf{r}_{M R_{2}}-{ }_{M} \mathbf{r}_{M R_{1}}\right\|_{2}$. The five dimensional unobservable sub-space of $J 3$ gets spanned by ${ }_{M} \boldsymbol{i},{ }_{M} \mathbf{r}_{M R_{i}}$ and $k_{m_{i}}$ and shows as increase of the spread. The solid lines are the mean, the shaded areas the standard deviation, and the dashed lines the ground truth of the respective estimates over 30 test runs. Axis x (blue), y (red), z (green) or rotor 1 (blue), 2 (red), 3 (green), 4 (orange).
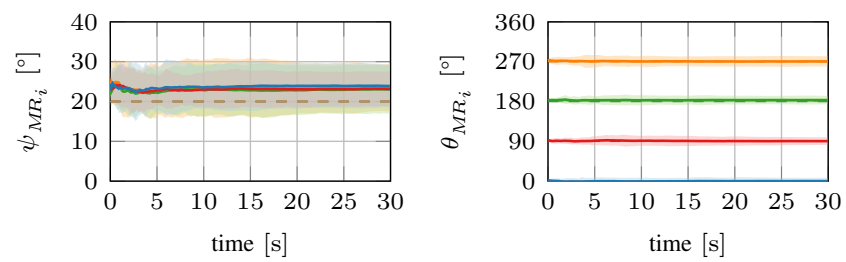

Fig. 6. Observable rotor axis angles $\theta_{M R_{i}}$ and $\psi_{M R_{i}}$ with non-zero $\psi_{M R_{i}}$. The solid lines are the mean, the shaded areas the standard deviation, and the dashed lines the ground truth of the respective estimates over 30 test runs. Axis x (blue), y (red), z (green) or rotor 1 (blue), 2 (red), 3 (green), 4 (orange). 


\section{ACKNOWLEDGMENTS}

Part of this work has received funding from the European Union's Horizon 2020 research and innovation programme under grant agreement 871260 .

\section{REFERENCES}

[1] C. Böhm, G. Li, G. Loianno, and S. Weiss. ObservabilityAware Trajectories for Geometric and Inertial SelfCalibration. In Power On and Go Robots 2020, RSS'20, (Virtual) Workshop, July 2020.

[2] M. Burri, J. Nikolic, H. Oleynikova, M. W. Achtelik, and R. Siegwart. Maximum Likelihood Parameter Identification for MAVs. In 2016 IEEE International Conference on Robotics and Automation (ICRA), pages 4297-4303, May 2016.

[3] M. Burri, M. Bloesch, Z. Taylor, R. Siegwart, and J. Nieto. A framework for maximum likelihood parameter identification applied on MAVs. Journal of Field Robotics (JFR), 35(1):5-22, January 2018.

[4] M. Dhaybi and N. Daher. Accurate Real-time Estimation of the Inertia Tensor of Package Delivery Quadrotors. In 2020 American Control Conference (ACC), pages 15201525, July 2020.

[5] F. Furrer, M. Burri, M. Achtelik, and R. Siegwart. RotorS - A Modular Gazebo MAV Simulator Framework. In Robot Operating System (ROS): The Complete Reference (Volume 1), pages 595-625. Springer International Publishing, 2016.

[6] K. Hausman, J. Preiss, G. S. Sukhatme, and S. Weiss. Observability-Aware Trajectory Optimization for SelfCalibration With Application to UAVs. IEEE Robotics and Automation Letters (RA-L), 2(3):1770-1777, July 2017.

[7] R. Hermann and A. Krener. Nonlinear controllability and observability. IEEE Transactions on Automatic Control (TAC), 22(5):728-740, October 1977.

[8] M. Kamel, T. Stastny, K. Alexis, and R. Siegwart. Model Predictive Control for Trajectory Tracking of Unmanned Aerial Vehicles Using Robot Operating System. In Robot Operating System (ROS): The Complete Reference (Volume 2), pages 3-39. Springer International Publishing, 2017.

[9] J. Kelly and G. S. Sukhatme. Visual-Inertial Sensor Fusion: Localization, Mapping and Sensor-to-Sensor Self-calibration. The International Journal of Robotics Research (IJRR), 30(1):56-79, January 2011.

[10] A. Martinelli. Continuous Symmetries and Observability Properties in Autonomous Navigation. Research Report RR-7049, INRIA, October 2010.

[11] D. Mellinger, Q. Lindsey, M. Shomin, and V. Kumar. Design, Modeling, Estimation and Control for Aerial Grasping and Manipulation. In 2011 IEEE/RSJ International Conference on Intelligent Robots and Systems (IROS), pages 2668-2673, September 2011.

[12] J. A. Preiss, K. Hausman, G. S. Sukhatme, and S. Weiss. Simultaneous self-calibration and navigation using trajec- tory optimization. The International Journal of Robotics Research (IJRR), 37(13-14):1573-1594, August 2018.

[13] D. Six, S. Briot, J. Erskine, and A. Chriette. Identification of the Propeller Coefficients and Dynamic Parameters of a Hovering Quadrotor From Flight Data. IEEE Robotics and Automation Letters (RA-L), 5(2):1063-1070, January 2020.

[14] J. Solà. Quaternion kinematics for the error-state Kalman filter. arXiv e-prints, arXiv:1711.02508, November 2017.

[15] J. Svacha, J. Paulos, G. Loianno, and V. Kumar. IMUBased Inertia Estimation for a Quadrotor Using NewtonEuler Dynamics. IEEE Robotics and Automation Letters (RA-L), 5(3):3861-3867, February 2020.

[16] N. Trawny and S. I. Roumeliotis. Indirect Kalman Filter for 3D Attitude Estimation. Technical Report 2005-002, MARS Lab, March 2005.

[17] D. Tzoumanikas, F. Graule, Q. Yan, D. Shah, M. Popović, and S. Leutenegger. Aerial Manipulation Using Hybrid Force and Position NMPC Applied to Aerial Writing. In Proceedings of Robotics: Science and Systems XVI. Robotics: Science and Systems Foundation, July 2020.

[18] D. Tzoumanikas, Q. Yan, and S. Leutenegger. Nonlinear MPC with Motor Failure Identification and Recovery for Safe and Aggressive Multicopter Flight. In 2020 IEEE International Conference on Robotics and Automation (ICRA), pages 8538-8544, August 2020.

[19] S. Weiss. Vision Based Navigation for Micro Helicopters. PhD thesis, ETH Zurich, 2012.

[20] S. Weiss and R. Siegwart. Real-Time Metric State Estimation for Modular Vision-Inertial Systems. In 2011 IEEE International Conference on Robotics and Automation (ICRA), pages 4531-4537, May 2011.

[21] V. Wuest, V. Kumar, and G. Loianno. Online Estimation of Geometric and Inertia Parameters for Multirotor Aerial Vehicles. In 2019 IEEE International Conference on Robotics and Automation (ICRA), pages 1884-1890, May 2019. 